\title{
PARTO HUMANIZADO: O PAPEL DA DOULA E A VISÃO DO ENFERMEIRO
}

\author{
HUMANIZED CHILD-BIRTH: THE ROLE OF THE DOULA AND THE \\ NURSE'S PERSPECTIVE
}

\author{
Maria Salete Vaceli Quintilio, Rafaella Barreto Borges de Castro, Jayne Lins de Souza
}

Faculdade Sena Aires - FACESA

\begin{abstract}
The humanization of Childbirth Care guarantees women emotional, cultural, educational and physical respect, where a 'doula' can be chosen as a companion before and during and after childbirth, offering the necessary assistance for the well-being of the pregnant woman and inducing a delivery faster and decreasing obstetric interventions. This study is a literature review that aims to identify the knowledge of nursing professionals about the doula in humanized childbirth. The research used free scientific databases, where works published in Portuguese between 2001 and 2020 were selected. This study points out that doulas can provide emotional support, physical and mental comfort, where non-pharmacological methods are used for relief pain and establishes a bond between the health team and the woman. However, for some health professionals, the work of the doula is unnecessary, as they recognize that the practices used are within the competence of nursing.
\end{abstract}

Keywords: Humanized birth; doulas; nurses; Perinatal care

\section{Resumo}

A humanização da Assistência ao Parto garante à mulher respeito emocional, cultural, educacional e físico, onde uma 'doula' pode ser escolhida como acompanhante antes, durante $e$ após o parto, oferecendo a assistência necessária para o bem-estar da gestante e induzindo um parto mais rápido e diminuindo intervenções obstétricas. Este estudo trata-se de uma revisão de literatura que tem o intuito de identificar $o$ conhecimento dos profissionais de enfermagem sobre a doula no parto humanizado. A pesquisa utilizou-se de bases de dados científicas gratuitas, onde foram selecionadas obras publicadas em português entre 2001 a 2020. Este estudo aponta que as doulas podem proporcionar um apoio emocional, conforto físico e mental, onde são utilizados métodos não-farmacológicos para alívio da dor e estabelece um vínculo entre a equipe de saúde e a mulher. No entanto, para alguns profissionais da saúde, o trabalho da doula é dispensável, pois reconhecem que as práticas utilizadas são de competência da enfermagem.

Palavras-chave: Parto humanizado; doulas; enfermeiros; Assistência perinatal. 
O Parto Humanizado $(\mathrm{PH})$ é um tema que tem se destacado na atualidade. A humanização busca promover assistência integral à parturiente através de ações que respeitem e atendam a mulher durante o Trabalho de Parto (TP), considerando os aspectos biológicos, psicológicos, afetivos e espirituais. ${ }^{1}$

Dentro de o ambiente hospitalar, é comum que a parturiente se sinta incapaz de tomar decisões acercadas práticas que devem ser adotadas durante o processo de parto, deixando de ser o ator principal deste processo. Assim, a humanização tem um foco na autonomia da parturiente, respeitando-a como um indivíduo dotado de valores e hábitos individuais. ${ }^{2,3}$

Com os avanços tecnológicos, certos procedimentos foram incluídos e facilitados na assistência obstétrica. Desse modo, alguns processos interventivos que outrora só eram realizados em casos específicos e/ou emergenciais, passaram a ser rotina durante o atendimento neonatal. ${ }^{3}$ Ao tratar o parto como um processo fisiológico natural, tem-se a possibilidade de diminuir intervenções médicas e cirúrgicas, promovendo a inclusão de atividades que reduzem o desconforto emocional e físico da parturiente. $^{1}$

Diante destas novas práticas humanizadas e humanistas, a literatura cientifica ressalta a importância do suporte físico, emocional e psíquico que a mulher deve receber antes, durante e após o TP. Tal assistência pode ser provida por profissionais de saúde, familiares, leigos, ou ainda, por acompanhantes capacitadas para este tipo de atendimento, denominadas doulas. O suporte prestado à mulher em TP pelas doulas, geralmente consiste em massagens, palavras de encorajamento, informações sobre a evolução do parto e suporte defensivo que visa atender os desejos da parturiente e seus familiares. ${ }^{3}$

Segundo a Organização Mundial de Saúde (OMS), doula é a profissional e/ou voluntária especializada em acompanhar e prestar assistência à mulher durante o processo de gestação, parto e pós-parto. A doula oferece apoio emocional, visando acalmar e reduzir a ansiedade esclarece possíveis dúvidas, traz conforto físico e aliviam a dor através métodos naturais, além de estabelecer um vínculo de confiança e credibilidade entre a parturiente e a equipe de saúde responsável pelo atendimento. ${ }^{6}$ No Brasil, a atividade da doula foi registrada pela primeira vez em 1997 no Hospital Sofia Feldman (Belo Horizonte - MG). ${ }^{7}$ Contudo, apesar de não haver números exatos, estima-se que no país existem aproximadamente cerca de três mil doulas atuantes. O Ministério do Trabalho (MT) atualizou, em janeiro de 2013, a Classificação Brasileira de Ocupações (CBO), onde foi inserida a ocupação de doula. De acordo com a CBO, estas não exercem procedimentos médicos ou de enfermagem e não oferecem risco a mulher durante a assistência de parto ${ }^{7,8}$. As doulas podem trabalhar em hospitais e/ou domicílios, podendo ser chamada a pedido da gestante ou atuando em parceria com a equipe médica. ${ }^{9}$

Entre os benefícios observados na atuação destas profissionais, estão: redução do tempo de evolução do parto, tranquilidade e segurança da parturiente, atenuação das dores durante o TP, maior índice de aleitamento materno exclusivo, menor número de relatos de Depressão PósParto (DPP) e menor índice de cesáreas. ${ }^{7,10}$

Entretanto, observa-se que a inserção das doulas dentro das maternidades não considerou a participação e aprovação dos profissionais tradicionalmente inseridos neste contexto. Esta omissão, por vezes, gera atritos com a equipe médica em relação ao papel e função das doulas durante o TP. Tal cenário abre margem para a resistência e possíveis conflitos, principalmente com relação às orientações oferecidas pelas doulas que muitas vezes vai na contramão das orientações biomédicas. ${ }^{6}$

Assim, apesar do importante serviço prestado pelas doulas, este ainda é desconhecido pela população, inclusive pelos profissionais da saúde, fazendo com que estudos científicos que abordam esse assunto sejam de grande interesse e relevância.

Este artigo de revisão, norteado pela necessidade de conhecimento acerca da percepção dos profissionais da enfermagem e das clientes sobre o trabalho das doulas na assistência à mulher nos processos de gestação, parto e pós-parto, tem o intuito de destacar o papel da doula no parto humanizado e a visão que o enfermeiro tem sobre esse papel, visto que a aceitação e conhecimento deste profissional é muito importante na aceitação das doulas.

\section{Metodologia}

Este trabalho foi desenhado como uma Revisão de Literatura, envolvendo pesquisa em materiais já publicados e editados sobre o tema. Durante a coleta de dados foram encontrados cerca de 50 artigos abordando a Doula. A busca foi norteada pelo título do artigo, assim como pelas 
informações constantes no resumo, selecionando-se aqueles que indicavam a relação das doulas com os profissionais de saúde, com a humanização do parto e a percepção das parturientes sobre a participação das doulas ao longo da gestação e do parto.

Esta Revisão Literária, que também é pode ser denominada Revisão Bibliográfica, utilizou como mecanismo para a seleção de obras, os critérios de inclusão, a saber: a existência dos seguintes descritores ou palavras-chave, vinculados aos DeCs (Descritores em ciências da saúde): Parto humanizado; doulas; enfermeiros; Assistência perinatal. Além disso, os artigos foram selecionados nas bases de dados, disponíveis na internet, SciELO (Scientific Eletronic Library Online), BDENF (Base de Dados em Enfermagem), LILACS (Literatura Latino-Americana e do Caribe em Ciências da Saúde) e BVS (Biblioteca Virtual em Saúde). Foram selecionadas obras publicadas entre 2001 a 2020, em língua portuguesa e na íntegra, tanto originais como revisões teóricas e estudos observacionais.

Neste estudo foram incluídas 32 obras que cumpriam com os critérios descritos acima.

\section{Resultados e Discussão}

O processo do parto passou por uma série de transformações ao longo dos séculos, resultando, entre outras coisas, na substituição do parto domiciliar para o parto em ambiente hospitalar. ${ }^{11}$ Em alguns casos, a mulher era internada precocemente sem acompanhantes e com pouco acesso às informações sobre os procedimentos médicos a serem prestados, e com isso, perdia sua autonomia e poder de decisão sobre aceitar ou recusar determinadas condutas. A fim de remediar tais práticas, foi publicado em 2000, pelo Ministério da Saúde (MS) a portaria/GM no 589, que visa assegurar a melhoria do acesso, cobertura e qualidade da assistência pré-natal, parto e puerpério às gestantes e ao recémnascido (RN). ${ }^{5}$

Para garantir que o processo de humanização do parto fosse respeitado foi, então, sancionada a Lei $\mathrm{n}$-11.108, que garante a presença de um acompanhante junto à mulher durante o TP. Com efeito, a parturiente poderá escolher qualquer profissional de saúde, acompanhante (doula) e familiar para lhe prestar assistência. ${ }^{12}$

No Brasil, existem cursos profissionalizantes de doula que visam preparar profissionais para exercer este papel de forma voluntária ou remunerada. ${ }^{13}$ Pesquisas internacionais discorrem sobre os benefícios do papel da doula no TP, apontando que as mulheres acompanhadas por tais profissionais/voluntários apresentaram maiores chances de ocorrer parto natural, sem intervenções, o que resulta em menores danos físicos, emocionais e psicológicos a mulher e ao recém-nascido ${ }^{7}$.

\section{A humanização do parto}

Atualmente a humanização da saúde tem sido abordada em muitas pesquisas enquanto subsídio para a consolidação dos valores e princípios do Sistema Único de Saúde (SUS). Humanizar significa, em termos gerais, focar o ser humano como sendo único, respeitando todos os seus processos de vivência social, emocional, psíquica, cultural e educacional. ${ }^{14}$

Assim, a humanização diz respeito à valorização das dimensões subjetiva e social do ser, abrangendo todas as práxis do SUS, garantindo o acesso às informações sobre a saúde pelos usuários e possibilitando o estabelecimento de vínculos solidários e de participação coletiva. ${ }^{15}$ Em 2003, foi criada a Política Nacional de Humanização (PNH), ou HumanizaSUS, uma política transversal de assistência à saúde com caráter coletivo, multiprofissional e dimensional. ${ }^{16}$

Desta forma, a Humanização da Assistência ao Parto (HAP) foi concebida como um dos braços da $\mathrm{PNH}$ a fim de assegurar à gestante o respeito a sua subjetividade, em uma fase da vida na qual ela precisa de atenção e cuidado. ${ }^{17}$ Assim, as práticas e intervenções dos profissionais de saúde devem ser executadas com o intuito de favorecer o processo do parto, o que dá à mulher o papel de protagonista neste cenário. ${ }^{18,19,20}$

Além do mais, a HAP visa garantir à parturiente alguns direitos, tais como: presença de acompanhante de sua escolha; autonomia para ser informada e solicitar sua permissão ante a prática de qualquer procedimento; ambiente acolhedor; contato e permanência de mãe-bebê. Ressalva-se que a humanização deve ocorrer independentemente do tipo de parto a ser realizado. $^{17}$

A presença de uma acompanhante que traga orientações, alívio das dores e tranquilidade à parturiente são reconhecidos pelo MS, sob a Lei 11.108/2005 que preconiza que as instituições de saúde permitam e estimulem a presença de um acompanhante durante todo o processo de préparto-pós. $^{21}$

Deste modo, a presença de uma acompanhante profissional é um diferencial oferecido à mulher durante a gestação, parto e pós-parto. A segurança do suporte ofertado durante o TP 
pode ser, segundo Leão e Bastos ${ }^{7}$, classificada da seguinte forma: emocional (palavras tranquilizantes e incentivos de encorajamento); física (massagens, compressas); informativa (orientações, instruções e conselhos) e defensiva (interpretar os desejos e anseios da mulher para os profissionais de saúde).

\section{A doula}

A palavra "doula" tem sua etiologia grega que significa "mulher que serve". Na segunda metade do século $\mathrm{XX}$, o termo doula foi resgatado pela antropóloga americana, Dana Raphael, para referir-se à companheira que oferta à mulher suporte emocional e físico durante o TP. 4, 5,10

Estas profissionais/voluntárias fazem uso durante sua atuação tanto da medicina alternativa e complementar, quanto da tradicional. A profissão de doula foi regularizada, no Brasil, pelo Ministério da Saúde, por meio da Portaria 971 que sancionou a Política Nacional de Práticas Integrativas Complementares (PICs) com vistas a garantir a prevenção de agravos, promoção e recuperação da saúde. ${ }^{19}$ As PICs têm por objetivo desenvolver na mulher a aquisição de competências de autocuidado e autoestima, assim como integrá-las ao meio ambiente e a sociedade. $^{22}$

A presença de uma acompanhante qualificada ao lado da parturiente traz conforto físico, segurança e encorajamento, o que reduz o nível de ansiedade da parturiente e ajuda-a a sentir-se em segurança, o que pode induzir um parto mais rápido e com menores índices de intervenções obstétricas. $^{7}$

E embora alguns estudos tragam evidências cientificas sobre os benefícios da presença das doulas durante o TP, estas ainda encontram resistência por parte de alguns profissionais e instituições, que as consideram acompanhantes, fazendo com que a mulher tenha que escolher entre as doulas e um familiar. No entanto, o projeto de lei $n$ 2195/13 visa fixar um piso salarial a esta categoria profissional e a garantia de sua permanência com a parturiente durante todo o processo de parto sem prejuízo a outro/a acompanhante à escolha da mulher. ${ }^{6,7}$

\section{A função da doula na assistência à mulher em trabalho de parto na perspectiva dos enfermeiros e profissionais da saúde}

A primeira competência das doulas apontada pelos profissionais da saúde diz respeito ao aspecto emocional da mulher durante o TP. Neste aspecto, as doulas entram em confrontos com a equipe de psicologia, que aponta os limites destas profissionais no âmbito do amparo emocional as parturientes.

$\mathrm{Na}$ percepção da equipe de saúde, segundo Herculano et al. $^{23}$, apenas uma minoria de profissionais considera as doulas como necessárias. Contudo, estudos comprovam a eficácia da doula ao manter a parturiente calma e tranquila em detrimento ao acompanhante (geralmente um familiar), haja vista a falta de vínculos afetivos entre doula-parturiente. ${ }^{24}$

A segunda competência apontada refere-se aos aspectos físicos na qual as doulas utilizam métodos não-tradicionais (ou nãofarmacológicos) para alívio da dor, como massagens, exercícios, compressas e mudanças de posição. Desta forma, as doulas proporcionam à mulher que vivencie uma experiência de parto o mais positiva e natural possível. Considerandose o custo e baixos riscos, os métodos nãotradicionais são defendidos e estimulados pela PNH para serem utilizados pela equipe de saúde. $^{23,25}$

A terceira competência apontada pelos profissionais de saúde, diz respeito ao suporte à informação, com a qual a doula deve orientar o processo do TP discorrendo sobre a fisiologia dos eventos ocorridos. Estas informações são apontadas pelos profissionais como um instrumento para facilitar e desmistificar a aceitação do Parto Normal (PN). ${ }^{23}$

\section{A relação da doula com os profissionais de saúde}

Para os profissionais que compõem a equipe de enfermagem e atuam na assistência ao préparto, parto e pós-parto, há uma carência de profissionais especializados e capacitados a atenderem a mulher durante este processo. Segundo Herculano e colaboradores ${ }^{23}$, os profissionais de enfermagem reconhecem que a assistência prestada pelas doulas durante o TP é de competência da enfermagem. Contudo, devido à sobrecarga de trabalho e o déficit de profissionais da categoria, tal assistência é fornecida de forma deficiente e/ou ineficaz pela equipe de enfermagem, daí a importância das doulas para esta categoria profissional, visto que estas dedicam-se, exclusivamente, às parturientes e suas necessidades.

Deste modo, a atividade da doula traz certa tranquilidade à equipe, pois elas informam qualquer necessidade da gestante. Contudo, mesmo com essa prerrogativa, a categoria de enfermagem aponta a interferência das doulas em condutas dos profissionais de enfermagem como ponto de conflito. ${ }^{23,27}$ 
Frente a este cenário, o movimento de humanização propõe um modelo colaborativo de cuidado entre a equipe de saúde e as doulas para condução dos partos de risco. ${ }^{11}$

Portela et al. $^{28}$ afirma que um modelo colaborativo na assistência obstétrica brasileira ainda está distante de ser sedimentado. De acordo com os autores, há alguns desafios para a efetivação deste modelo, como a dificuldade de redistribuição de poder, a construção de relações de confiança entre a equipe e por fim, a desmistificação da intervenção profissional.

\section{Doula e a percepção das parturientes}

O parto é um dos momentos mais importantes na vida de uma mãe, um instante único, sendo assim, elas esperam uma atenção total de toda equipe multidisciplinar, principalmente da enfermagem. No TP mulheres tem a expectativa que a enfermagem obstétrica doe todo o seu tempo, suporte físico e psicológico, mas o tempo dedicado chega a ser mínimo devido à sobrecarga do trabalho dos profissionais de enfermagem. ${ }^{31}$ Tendo em vista essa escassez de atenção, a Doula substitui essa falta e proporciona a atenção e os cuidados necessário a mãe.

A sobrecarga de trabalho da enfermagem interfere na troca de informações durante o prénatal. Estudos mostram que há falta de conhecimento das mães em relação as doulas, tendo observado que gestantes multíparas não obtiveram nenhumas informações durante suas consultas de pré-natal. ${ }^{33}$ Algumas até ouviram comentários sobre Doula, mas não tinham informações o suficiente sobre o que realmente seria uma Doula.

Segundo uma pesquisa realizada com algumas mulheres que tiveram a presença de Doulas ao longa da gestação e durante o parto, foi relatado a satisfação e o conforto transmitido pela doula, tendo como benefícios menor tempo de parto, menor taxa de intervenções hospitalares como anestesias e cesárias. ${ }^{30}$ Sendo assim, a presença das Doulas foi avaliada de forma positiva entre as parturientes.

Contudo foi ressaltado que por mais que a presença da doula seja avaliada de forma positiva, não é o suficiente para que haja um parto humanizado, pois há outros diversos problemas sendo um deles o equilíbrio entre um bom ambiente hospitalar e a sintonia entre a equipe multidisciplinar, a instituição e a Doula. ${ }^{30}$

\section{Considerações finais}

Este estudo destacou que a presença da doula pode oferecer à parturiente um parto mais seguro e natural, com a utilização de métodos não-farmacológicos, obtendo assim menores índices de intervenção obstétrica. Aos profissionais de saúde, a doula pode auxiliar na diminuição da sobrecarga de trabalho, pois pode se encarregar de vários procedimentos, proporcionando condições para que o profissional da saúde tenha um melhor desempenho em suas funções específicas e assegurando um parto humanizado.

A percepção dos profissionais da saúde e enfermeiros sobre o papel da doula ainda é deficiente em informações e reconhecimento. Apenas uma minoria considera a doula como uma profissional capacitada e necessária, sendo que o restante acredita que o serviço prestado pelas doulas é de competência da enfermagem, opinião que gera conflitos entre ambos. Portanto, existe uma necessidade premente da construção de relações de confiança e troca de informações dentro deste contexto.

Desta forma, pode-se concluir que, apesar das doulas prestarem um serviço benéfico às gestantes e parturientes e, consequentemente, auxiliarem a equipe de saúde atuante neste cenário, seu papel ainda não é reconhecido e/ou bem aceito pela comunidade de profissionais da saúde. Este fato, aliado à escassez de conhecimento acerca das doulas e sua influência no panorama da saúde no Brasil, indica uma necessidade de mais estudos e artigos que abordem estes temas.

\section{Referências}

1. Gonçalves $\mathrm{R}$, et al. Vivenciando $\mathrm{o}$ cuidado no contexto de uma casa de parto: o olhar das usuárias. Rev. Esc. Enferm. Usp. 2011; 45(1): 62-70.

2. Dias MAB, Domingues RMSM. Desafios na implantação de uma política de humanização da assistência hospitalar ao parto. Ciênc. Saúde coletiva. 2005;10(3):669-705.

3. Castro JC de,Clapis MJ. Parto humanizado na percepção das enfermeiras obstétricas envolvidas com a assistência ao parto. Rev. latinoamenferm. 2005;13(6):960-67.

4. Hogab LAK, Pinto CM de S. Assistência ao parto com a presença do acompanhante: Experiências de profissionais. Invest. educ. enferm. 2007; 25(1);74-81.

5. Leão VM, Oliveira SMJV. O papel da doula na assistência à parturiente. Rev. Min. Enf. 
[periódico da internet] 2005; 10(1):24-9. Acesso às 17:00h de 02/06/2020. Disponível em: http://www.portalbvsenf.eerp.usp.br/scielo.php ?script=sci arttext\&pid=S1415$\underline{27622006000100005 \& \operatorname{lng}=e s \& n r m=i s o}$

6. Santos DS, Nunes IM. Doulas na Assistência ao Parto: Concepção de Profissionais de Enfermagem. Esc Anna Nery. 2009; 13(3):582588.

7. LEÃO, M. R. C.; BASTOS, M. A. R. Doulas apoiando mulheres durante o trabalho de parto: experiência do Hospital Sofia Feldman. Rev Latino-am Enfermagem, v. 9, n. 3, p. 90-4, 2001.

8. DUARTE, A. C.. Doula a quem doer. O Estado de S. Paulo, São Paulo. 02 fev. 2013. Acesso às 15:00h de 02/06/2020. Disponível em: http://www.estadao.com.br/noticias/suplement os,doula-a-quemdoer, $992218,0 . \mathrm{htm}$

9. Basevi A. Quem é a Doula? 2004. Acesso às 15:00h de 04/06/2020. Disponível em: www.amigasdoparto.com.br

10. Duarte, A C. Quem são as Doulas. 2007. Acesso às 11:00h de 02/06/2020. Disponível em: http://www.doulas.com.br/

11. Rodrigues AV, Siqueira AF. Sobre as dores e temores do parto: dimensões de uma escuta. Rev. Bras. Saúde Matern Infantil 2008; 8(2):179-186.

12. Brasil. Lei no 11.108 , de 08 de abril de 2005. Altera a Lei n. 8.080, de 19 de setembro de 1990, para garantir às parturientes o direito à presença de acompanhante durante o trabalho de parto, parto e pósparto imediato, no âmbito do Sistema Único de Saúde - SUS. Diário Oficial da República 2005; 8 abr.

13. Doulas no Brasil. [site da internet] 2020. Acesso às 15:00h de 05/06/2020. Disponível em: http://www.doulas.com.br.

14. RIBEIRO MILC, FUREGATO ARF. Reflexões sobre a importância do relacionamento interpessoal na formação de profissionais de enfermagem. Nursing (São Paulo). 2003;6(66):19-24.

15. REGO $S$, GOMES AP, BATISTA RS. Bioética e humanização como temas transversais na formação médica. RevBrasEduc Med. 2008;32(4):482-91.

16. MINISTÉRIO DA SAÚDE. Programa Nacional de Humanização da Assistência Hospitalar. Brasília (Brasil): Ministério da Saúde; 2001.

17. Brasil. Ministério Público de Pernambuco. (2015). Humanização do Parto Nasce o Respeito: Informações práticas sobre seus direitos. Recife, PE: Procuradoria Geral de Justiça. Acesso às 15:00h de 05/06/2020. Disponível em:http://www.mppe.mp.br/mppe/attachments Larticle/4240/cartilha\%20\%20humanizacao\%20d o\%20parto\%20pdf.pdf

18. Ferreira Junior, A. R., \& Barros, N. F. (2016). Motivos para atuação e formação profissional: percepção de doulas. Physis Revista de Saúde Coletiva, 26(4), 1395-1407. Acesso às 11:00h de 05/06/2020. Disponível em: https://www.scielo.br/scielo.php?script=sci_artt ext\&pid=S0103-73312016000401395

19. Barros, N. F. (2006). Política Nacional de Práticas Integrativas e Complementares no SUS: uma ação de inclusão. Ciência \& Saúde Coletiva, 11(3), 850-850. . Acesso às 11:00h de 05/06/2020. Disponível em: https://www.scielo.br/scielo.php?pid=S1413$81232006000300034 \&$ script $=$ sci arttext

20. Barros, L. P., Souza, C. L. T., Gonçalves, L. F., Gonzaga, L. N., Paula, T. A., \& Silva, A. M. (2015). O parto humanizado e o seu impacto na assistência a saúde. Revista Educação em Saúde, $3(2), 64-71$. Acesso às $19: 00 \mathrm{~h}$ de 05/06/2020. Disponível em: http://periodicos.unievangelica.edu.br/index.php \%20educacaoemsaude/article/view/1387/1271 21. Oliveira, A. S. S., Rodrigues, D. P., Guedes, M. V. C., Felipe, G. F., Galiza, F.T., \& Monteiro, L.C. (2011). O acompanhante no momento do trabalho de parto e parto: percepção de puérperas. Cogitare Enfermagem, 16 (2), 247-253.Acesso às 15:00h de 12/06/2020. Disponível em: https://revistas.ufpr.br/cogitare/article/view/202 $\underline{01}$

22. Telesi Júnior, E. (2016). Práticas integrativas e complementares em saúde, uma nova eficácia para o SUS. Estudos avançados, $30(86), 99-112$. Acesso às $15: 00 h$ de 16/06/2020. Disponível em: https://www.scielo.br/scielo.php?script=sci artt ext\&pid=S0103-40142016000100099

23. Herculano TB, et al. Doulas como gatilho de tensões entre modelos de assistência obstétrica: o olhar dos profissionais envolvidos. Saúde Debate | Rio De Janeiro, V. 42, N. 118, P. 702-713, jul-set 2018

24. Simas R. Doulas e o movimento pela humanização do parto: poder, gênero e a retórica do controle das emoções [dissertação]. Niterói: Universidade Federal Fluminense; 2016.

25. Mafetoni RR, Shimo AKK. Métodos não farmacológicos para alívio da dor no trabalho de parto: revisão integrativa. REME Rev Min Enferm. 2014; 18(2):505-520.

26. Simas R, Mendonça SS. O caso Adelir e o movimento pela humanização do parto: 
reflexões sobre violência, poder e direito. Vivência: Rev Antropol. 2017; 1(48):89-103.

27. Brasil. Ministério da Saúde. Caderno Humaniza SUS: humanização do parto e do nascimento. Brasília, DF: MS; 2014.

28. Portela MC, Reis LGC, Martins M, et al. Cuidado obstétrico: desafios para melhoria da qualidade. Cad Saúde Pública. 2018; 34(5):1-3.

29. MINAYO MCS. Ciência, técnica e arte: o desafio da pesquisa social. In: Minayo MCS, editor. Pesquisa social: teoria, método e criatividade. 23a ed. Petrópolis: Vozes; 2004. p. 9 $-29$.

30. ALMEIDA, Ana Claudia Santos et al. A percepção das gestantes sobre a atuação das doulas no parto natural.

31. LEÃO, Viviane Murilla; OLIVEIRA, Sonia Maria Junqueira Vasconcellos de. O papel da doula na assistência à parturiente. Revista Mineira de Enfermagem, v. 10, n. 1, p. 24-29, 2006.

32. SILVA, Lorena Carla Cardoso; CORRÊACUNHA, Elza Francisca; KAPPLER, Stella Rabello. Percepção de mulheres sobre o parto e o papel da doula. Psicologia Revista, v. 27, n. 2, p. $357-$ $376,2018$. 


\section{Endereço para Correspondência}

Maria Salete Vaceli Quintilio

Condomínio Belvedere Green, conj.3, cs 12 - Jd.

Botãnico - Brasília-DF

E-mail: saletevaceli@senaaires.com.br

Recebido em 26/05/2021

Aprovado em 15/09/2021

Publicado em 30/12/2021 